American Journal of Immunology 3 (1): 10-14, 2007

ISSN 1553-6203

(C) 2007 Science Publications

\title{
The Pathology of Alzheimer Disease Elicits an In Vivo Immunological Response
}

\author{
${ }^{1}$ David J. Bonda, ${ }^{1}$ Kate M. Webber, ${ }^{1}$ Sandra L. Siedlak, \\ ${ }^{1,2}$ George Perry, ${ }^{3}$ Robert P. Friedland and ${ }^{1}$ Mark A. Smith \\ ${ }^{1}$ Department of Pathology, Case Western Reserve University, Cleveland, Ohio, USA \\ ${ }^{2}$ College of Sciences, University of Texas at San Antonio, San Antonio, Texas, USA \\ ${ }^{3}$ Department of Neurology, Case Western Reserve University, Cleveland, Ohio, USA
}

\begin{abstract}
The mechanism(s) responsible for the loss of neurons that characterizes Alzheimer disease is incompletely understood. Nonetheless, there is considerable evidence suggestive of immune abnormalities coupled to alterations in blood-brain barrier permeability that likely play a key role in both the etiology and progression of the disease. To examine these issues further, this study was designed to examine the presence of human antibodies within hippocampal regions of both diseased and normal brains. Specifically, using antibodies directed against either human lambda $(\lambda)$ or kappa $(\kappa)$ subunits of human IgG, we examined the amount and localization of endogenous human antibodies within the brain. In cases of Alzheimer disease, but not in age-matched controls, we found human antibodies associated with pyramidal neurons and dystrophic neurites surrounding amyloid plaques pathological structures that characterize the disease. Since such human immunoglobulins likely originate in the vasculature, we also examined cases of cerebral amyloid angiopathy to further explore the importance of blood-brain barrier breaches and found high levels of antibodies associated with many blood vessels as well as pyramidal neurons. Taken together, these findings strengthen the notion that alterations in blood brain barrier permeability in both Alzheimer disease and cerebral amyloid angiopathy leads to the accumulation of antibodies that then may contribute to the inflammatory cascade within the brain.
\end{abstract}

Key words: Alzheimer disease, amyloid angiopathy, blood brain barrier, human immunoglobulin

\section{INTRODUCTION}

Alzheimer disease (AD) is a progressive and fatal neurodegenerative disease that is clinically characterized by dementia and neurobehavioral deterioration $^{[1-3]}$. Although the causes of the disease are not fully understood, many of its hallmark features are well known; such aspects include amyloid- $\beta$ senile plaques, neurofibrillary tangles (NFTs), degeneration of synapses, loss of neurons and cerebral amyloid angiopathy $(\mathrm{CAA})^{[4-11]}$. While the mechanisms responsible for this plethora of changes are incompletely understood, it is apparent that changes in blood-brain barrier (BBB) permeability coupled with inflammatory processes play a key role in disease development and progression.

The BBB provides the brain with a separation from the circulating blood of the body. Optimal functioning of the brain requires a specific homeostasis that would be disrupted by the multitude of chemicals and proteins within the blood, and the BBB protects this homeostasis by restricting the access of these compounds into the $\mathrm{CSF}^{[12]}$. A breach in the $\mathrm{BBB}$, as a result of disease or interstitial fluid of the brain injury, affords potentially damaging proteins access to the brain and causes damage and inflammation ${ }^{[12.13]}$. In $\mathrm{AD}$, studies have shown that non-steroidal anti-inflammatory drugs (NSAIDs) reduce the risk of disease ${ }^{[13]}$ and it is generally thought that inflammation plays a key role in the pathology. The relationship between inflammation and BBB dysfunction, however, remains unclear.

The human immune system is reliant upon the secretion and functioning of antigen-binding antibodies within the bloodstream. Each type of antibody, although unique to a particular antigen, is composed of two heavy and light polypeptide chains that define its class and subclass respectively. The heavy chains characterize the immunoglobulin ( $\mathrm{Ig}$ ) in one of five classes based on the polypeptide sequence of their constant regions: $\operatorname{IgA}$, $\operatorname{IgD}, \operatorname{IgE}, \operatorname{IgG}$ and $\operatorname{IgM}$. Similarly, the subclass of an Ig is governed by the constant region of its light chains and is described as

Corresponding Author: $\quad$ Mark A. Smith, Ph.D., Department of Pathology, Case Western Reserve University, 2103 Cornell Road, Cleveland, Ohio 44106 USA 
either human $\lambda$ or human $\kappa$. Therefore, although the variable regions of different antibodies are unique, the general presence of Igs can be detected via their constant regions (i.e., the light or heavy chain polypeptide sequence).

Under normal circumstances, these human antibodies have limited access to the CSF and are not involved in neuronal function. However, a breach in the BBB could enable the entrance of antibodies into the brain and result in neuroinflammation and degeneration. To further explore whether BBB changes might lead to permeability to serum antibodies, in this study we used antisera directed against either human $\operatorname{IgG} \lambda$ or $\kappa$ to detect the presence of human antibodies within the brain in cases of $\mathrm{AD}$ as well as in cases of severe CAA.

\section{MATERIALS AND METHODS}

Cases and neuropathologic assessment: Paraffin embedded tissue sections of human hippocampus were obtained from $11 \mathrm{AD}$ and 7 age-matched, nondemented 'control' cases. All samples were acquired from the Alzheimer Disease Research Center at Case Western Reserve University and fixed in either methacarn (methanol: chloroform: acetic acid; 6:3:1) or routine formalin. $\mathrm{AD}$ cases were confirmed pathologically and met CERAD criteria for neuropathologic diagnosis of $\mathrm{AD}^{[14,15]}$. $\mathrm{AD}$ cases ranged in age from 77 years to 87 years (mean 81) and had an average postmortem interval of four hrs. Likewise, control cases ranged in age from 66 to 82 years (mean 74). In addition, three aged cases of CAA, formalin fixed, were examined.

Immunocytochemistry: Sections were stripped of paraffin embedding using xylene, twice for $10 \mathrm{~min}$ at room temperature and rehydrated in descending concentrations of ethanol for ten min each at room temperature. Endogenous peroxidase activity was reduced by 30 min incubation in $3 \% \mathrm{H}_{2} \mathrm{O}_{2}$ in methanol. Subsequently, non specific binding sites were blocked with 10 min incubation in $10 \%$ normal goat serum (NGS) in Tris-buffered Saline (TBS; $50 \mathrm{mM}$ Tris-HCL and $150 \mathrm{mM} \mathrm{NaCl}, \mathrm{pH} 7.6)$. Adjacent tissue sections on each slide were incubated in a 1:50 dilution of either goat anti-human $\lambda$ or $\kappa$ antibody conjugated to horseradish peroxidase (Southern Biotech Birmingham, $\mathrm{AL}$ ) in TBS for $1 \mathrm{hr}$ at room temperature. Sections were then rinsed in $1 \%$ NGS in TBS solution and incubated in Tris buffer $(50 \mathrm{mM}, \mathrm{pH} 7.6)$ for $10 \mathrm{~min}$ at room temperature. Antibody binding sites were finally

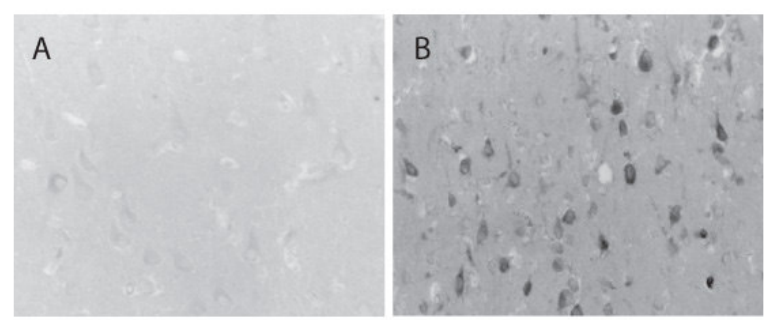

Fig. 1: $\quad$ AD hippocampal tissue sections demonstrate significant staining of neurons with anti-human $\lambda$ antibodies. $A$. In an $80 \mathrm{yr}$ old control case, neurons in the hippocampus demonstrate nominal staining with anti-human $\lambda$ antibodies. B. In a representative AD case, age 77, hippocampal neurons reveal striking labeling by human $\lambda$ antibodies.

detected using the chromagen $0.75 \mathrm{mg} / \mathrm{mL} \quad 3,3^{\prime}$ diaminobenzidine (DAB) with $0.015 \%$ hydrogen peroxide in Tris buffer. All slides were then dehydrated and mounted.

For cases of CAA, adjacent sections were immunostained with a monoclonal anti-amyloid antibody (4G8, Endogen) following a 70\% formic acid pretreatment for $5 \mathrm{~min}$. Development was performed using the peroxidase anti-peroxidase method with $\mathrm{DAB}$ as previously described ${ }^{[16,17]}$.

\section{RESULTS}

In approximately $80 \%$ of the AD cases (9 out of $11)$, there was marked immunostaining with goat antihuman $\lambda$ and $\kappa$ antibodies in the brain. Specifically, human $\lambda$ and $\kappa$ antibodies were detected in pyramidal neurons, senile plaque neurites and in association with blood vessels. By marked contrast, non-demented control cases demonstrated little or no staining. Figure 1 shows the marked difference between control (Fig. $1 \mathrm{~A})$ and $\mathrm{AD}$ (Fig. 1B) within pyramidal neurons. Figure 2 shows human $\lambda$ antibody in dystrophic neurites around amyloid- $\beta$ senile plaques in the AD cases. No differences in staining pattern or intensity were noted between $\lambda$ and $\kappa$ specific antibodies or between cases fixed with either formalin or methacarn. Pretreatments with either formic acid or other antigen retrieval techniques demonstrated equally effective labeling as native $\mathrm{AD}$ sections suggesting that there is no significant antigen masking effects.

To further explore the role of $\mathrm{BBB}$ breaches, cases of severe CAA were also examined. Like AD, the CAA cases demonstrated significant amounts of human immunoglobulins (both $\lambda$ and $\kappa$ ) within cerebral vessels (Fig. 3A) as well as within pyramidal neurons (not shown). Notably, there was only slight co-localization 


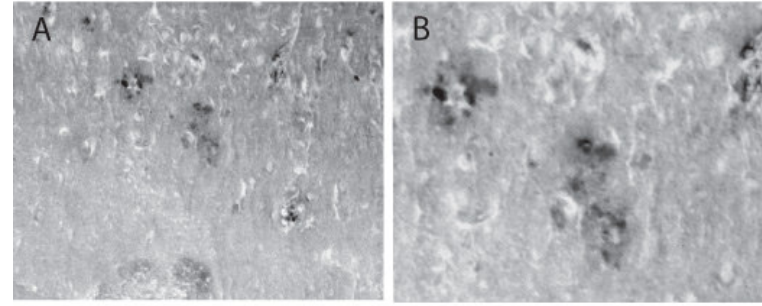

Fig. 2: Human $\lambda$ antibodies are localized to AD-related amyloid plaques. A. A representative $\mathrm{AD}$ case, age 88 , demonstrating amyloid plaque with positive stain of antihuman $\lambda$ antibody. $B$. Higher magnification of the structures in $A$.

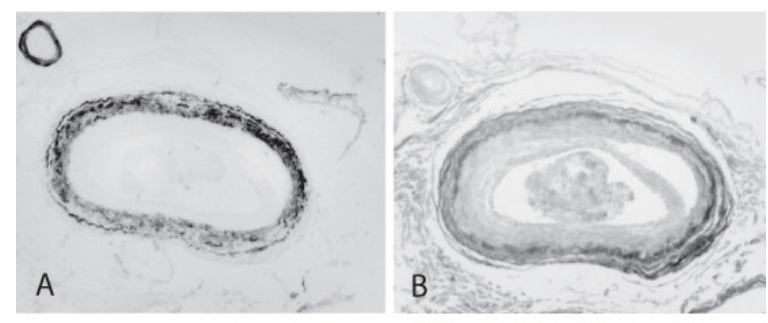

Fig. 3: Adjacent serial sections stained to compare the accumulation of human $\lambda$ antibodies with amyloid deposition with the vasculature of a case with severe CAA. In cases with severe CAA, many vessels accumulate amyloid $(A)$. As seen in the same field on an adjacent section, human $\lambda$ antibodies $(B)$ accumulate within some of the same vessels as amyloid. However, not all vessels with amyloid display human $\lambda$ antibody reactivity.

of human antibodies with the deposits of amyloid in blood vessels (Fig. 3 A,B).

\section{DISCUSSION}

In this study, we found human immunoglobulin subunits $\lambda$ and $\kappa$ associated with the pathology of AD. Specifically, pyramidal neurons, senile plaque, neurites and cerebral blood vessels all show marked elevations in human immunoglobulin as compared to agedmatched controls. Since the brain is classically considered to be immunologically privileged, these findings suggest that in $\mathrm{AD}$, there is a breach of this privilege, likely as a consequence of BBB breakdown and increased permeability. Indeed, the fact that $>80 \%$ of our $\mathrm{AD}$ cases show human IgG in brain is consistent with the proportion of $\mathrm{AD}$ cases estimated to have gross BBB alterations ${ }^{[18,19]}$.

The importance of $\mathrm{BBB}$ changes to our observations is also indicated by cerebral immunoglobulins within pyramidal neurons and the vasculature in cases of CAA. Notably, with the vessels of CAA cases, there was little relation between human immunoglobulins and amyloid- $\beta$ which is consistent with the notion that amyloid- $\beta$ occurs as a consequence to changes in the BBB rather than vice versa ${ }^{[17,20]}$. Our data thus suggests that human IgG may be a reliable and accurate indicator of BBB breaches.

The data acquired in this study reveal a significant association between the pathology typical of AD and human antibodies. Co-localization of human $\lambda$ and $\kappa$ type antibodies with amyloid plaques and the pyramidal neuron population susceptible to NFT development suggests a cause/effect relationship between the two and a potential link to neurodegeneration, although the exact connections remain uncertain. In particular, the role of the immune system in the disease, evident through the presence of the antibodies, cannot be accurately assessed. Previous studies have identified the presence of human antibodies in regions near amyloid deposition ${ }^{[21]}$. This study further promotes the pivotal role of the human immune system in $\mathrm{AD}$ and specifically infers an autoimmune characteristic to the disease.

One possibility for the role of the human immune system in the disease is that it functions as a response to the neuronal stress and inflammation instigated by amyloid- $\beta$ deposition and tau NFT development. This hypothesis presupposes that the pathological entities precede the generation and localization of the antibodies and that the antibodies are the body's healthy attempt to prevent further degeneration. However, previous studies have indicated the presence of antibodies in patients prior to development of pathology. Antimicroglial antibodies have been found in the cerebrospinal fluid in $\mathrm{AD}^{[22]}$. These studies demonstrate the presence of antibodies prior to significant inflammation.

Similarly, studies which describe evidence of antibodies in neurons that are otherwise healthy infer that they are a precedent to $\mathrm{AD}$ pathology ${ }^{[23]}$. Interestingly, seemingly "healthy" neurons within the hippocampal neuronal population, especially those susceptible to NFT development, have been shown to accumulate markers of oxidative damage well before the onset of AD symptoms or pathological development as well ${ }^{[24-27]}$. Specifically, increases in 8hydroxyguanosine and the lipid peroxidation product 4hydroxynonenal are considered markers of $\mathrm{AD}$ that accumulate within neurons before the appearance of NFTs ${ }^{[25.28,29]}$.

Serum levels of antibodies toward amyloid- $\beta$ have also been studied and have concluded very little distinction between levels in $\mathrm{AD}$ patients as compared to those of controls. Evidence even suggests lower levels of amyloid antibodies in serum of AD patients as 
compared to normal controls ${ }^{[30]}$. Consequently, although there are high concentrations of human antibodies within the brains in AD patients as revealed in this study, they do not appear to be specifically directed against the amyloid- $\beta$ epitope. This discovery leaves potential for the negative role of the immune system in the disease. Specifically, the antibodies in brain tissue of $\mathrm{AD}$ patients, as they both precede and are unrelated to the pathology of the disease, may, in fact, be attacking the tissue itself and be causing the characteristic inflammation of AD. Evidence even shows that immunoglobulin penetration into brain tissue results in a series of damaging consequences including demyelination, disruption of neural transmission, and cell death ${ }^{[31-33]}$ and such consequences directly apply to the pathogenesis of $\mathrm{AD}$, particularly to the overall neurodegeneration.

In summary, human antibody accumulation within susceptible neuronal populations, as well as neuritic plaques in tissue sections of AD brains in this study, characterize $\mathrm{AD}$ as an inflammatory immune disease. BBB dysfunction has been documented in cases of $\mathrm{AD}^{[34]}$ and would account for the unusual influx of human antibodies in brain tissue and lead to the ultimate demise of neurons. As evidenced by the notable accumulation of antibodies within many of the vessels in the cases of CAA shown in this study, a BBB disruption may in fact play a relevant role in the progression of the disease as well.

\section{ACKNOWLEDGMENTS}

Work in the authors' laboratories is supported in part by the National Institutes of Health (AG026151 to MAS; AG017173 to RPF), the Alzheimer's Association (GP), Philip Morris USA Inc. and Philip Morris International (MAS, GP), the Institute for the Study of Aging (RPF), the Joseph and Florence Mandel Research Fund (RPF), Pfizer (RPF), Eisai (RPF), and Forest Pharmaceuticals (RPF).

\section{REFERENCES}

1. Folstein, M.F. and F.W. Bylsma, 1999. Noncognitive Symptoms of Alzheimer Disease. In: Alzheimer Disease (Eds. R.D. Terry, R. Katzman, K.L. Bick and S.S. Sisodia) pp. 25-37. Lippincott Williams \& Wilkins, Philadelphia.

2. Morris, J.C., 1999. Clinical Presentation and Course of Alzheimer Disease. In: Alzheimer Disease (Eds. R.D. Terry, R. Katzman, K.L. Bick and S.S. Sisodia) pp. 11-24. Lippincott Williams \& Wilkins, Philadelphia.
3. West, M.J., C.H. Kawas, W.F. Stewart, G.L. Rudow and J.C. Troncoso, 2004. Hippocampal neurons in pre-clinical Alzheimer's disease. Neurobiol. Aging, 25: 1205-1212.

4. Smith, M.A., 1998. Alzheimer disease. Int. Rev. Neurobiol., 42: 1-54.

5. Terry, R.D., E. Masliah and L. Hansen, 1999. The Neuropathology of Alzheimer Disease and the Structural Basis of its Cognitive Alterations. In: Alzheimer disease (Eds. R.D. Terry, R. Katzman, K.L. Bick and S.S. Sisodia) pp: 187-206. Lippincott Williams \& Wilkins, Philadelphia.

6. Castellani, R.J., M.A. Smith, G. Perry and R.P. Friedland, 2004. Cerebral amyloid angiopathy: major contributor or decorative response to Alzheimer's disease pathogenesis. Neurobiol. Aging, 25: 599-602; discussion 603-594.

7. West, M.J., P.D. Coleman, D.G. Flood and J.C. Troncoso, 1994. Differences in the pattern of hippocampal neuronal loss in normal ageing and Alzheimer's disease. Lancet, 344: 769-772.

8. Gomez-Isla, T., J.L. Price, D.W. McKeel, Jr., J.C. Morris, J.H. Growdon and B.T. Hyman, 1996. Profound loss of layer II entorhinal cortex neurons occurs in very mild Alzheimer's disease. J. Neurosci., 16: 4491-4500.

9. Price, J.L., A.I. Ko, M.J. Wade, S.K. Tsou, D.W. McKeel and J.C. Morris, 2001. Neuron number in the entorhinal cortex and CA1 in preclinical Alzheimer disease. Arch. Neurol., 58: 1395-1402.

10. Sillus, M., W. Saeger, R.P. Linke, D. Muller and C. Voigt, 1993. Cerebral amyloid angiopathy. Frequency, significance and immunohistochemistry. Zentralbl. Pathol., 139: 207-215.

11. Vinters, H.V., W.M. Pardridge, D.L. Secor and N. Ishii, 1988. Immunohistochemical study of cerebral amyloid angiopathy. II. Enhancement of immunostaining using formic acid pretreatment of tissue sections. Am. J. Pathol., 133: 150-162.

12. de Vries, H.E., J. Kuiper, A.G. de Boer, T.J. Van Berkel and D.D. Breimer, 1997. The blood-brain barrier in neuroinflammatory diseases. Pharmacol. Rev., 49: 143-155.

13. Szekely, C.A., T. Town and P.P. Zandi, 2007. NSAIDs for the chemoprevention of Alzheimer's disease. Subcell. Biochem., 42: 229-248.

14. Khachaturian, Z.S., 1985. Diagnosis of Alzheimer's disease. Arch. Neurol., 42: 1097-1105.

15. Mirra, S.S., A. Heyman, D. McKeel, S.M. Sumi, B.J. Crain, L.M. Brownlee, F.S. Vogel, J.P. Hughes, G. van Belle and L. Berg, 1991. The Consortium to Establish a Registry for Alzheimer's Disease (CERAD). Part II. Standardization of the neuropathologic assessment of Alzheimer's disease. Neurology, 41: 479-486. 
16. Perry, G., M.A. Smith, C.E. McCann, S.L. Siedlak, P.K. Jones and R.P. Friedland, 1998. Cerebrovascular muscle atrophy is a feature of Alzheimer's disease. Brain Res., 791: 63-66.

17. Kawai, M., R.N. Kalaria, P. Cras, S.L. Siedlak, M.E. Velasco, E.R. Shelton, H.W. Chan, B.D. Greenberg and G. Perry, 1993. Degeneration of vascular muscle cells in cerebral amyloid angiopathy of Alzheimer disease. Brain Res., 623: 142-146.

18. Mandybur, T.I., 1975. The incidence of cerebral amyloid angiopathy in Alzheimer's disease. Neurology, 25: 120-126.

19. Glenner, G.G., J.H. Henry and S. Fujihara, 1981. Congophilic angiopathy in the pathogenesis of Alzheimer's degeneration. Ann. Pathol., 1: 120129.

20. Atwood, C.S., R.L. Bowen, M.A. Smith and G. Perry, 2003. Cerebrovascular requirement for sealant, anti-coagulant and remodeling molecules that allow for the maintenance of vascular integrity and blood supply. Brain Res. Brain Res. Rev., 43: 164-178.

21. D'Angelo, C. and G.B. D'Angelo, 1986. Autoantibodies similar to neurofilament antibodies, directed against tangles and senile plaques are present in patients with presenile and senile dementia or with Down's syndrome. EOS Riv. Immunol. Immunofarmacol. , 6: 8-12.

22. McRae, A., R.N. Martins, J. Fonte, R. Kraftsik, L. Hirt and J. Miklossy, 2007. Cerebrospinal fluid antimicroglial antibodies in Alzheimer disease: a putative marker of an ongoing inflammatory process. Exp. Gerontol., 42: 355-363.

23. D'Andrea, M.R., 2003. Evidence linking neuronal cell death to autoimmunity in Alzheimer's disease. Brain Res., 982: 19-30.

24. Nunomura, A., G. Perry, M.A. Pappolla, R. Wade, K. Hirai, S. Chiba and M.A. Smith, 1999. RNA oxidation is a prominent feature of vulnerable neurons in Alzheimer's disease. J. Neurosci., 19: 1959-1964.

25. Nunomura, A., G. Perry, G. Aliev, K. Hirai, A. Takeda, E.K. Balraj, P.K. Jones, H. Ghanbari, T. Wataya, S. Shimohama, S. Chiba, C.S. Atwood, R.B. Petersen and M.A. Smith, 2001. Oxidative damage is the earliest event in Alzheimer disease. J. Neuropathol. Exp. Neurol., 60: 759-767.
26. Castellani, R.J., P.L. Harris, L.M. Sayre, J. Fujii, N. Taniguchi, M.P. Vitek, H. Founds, C.S. Atwood, G. Perry and M.A. Smith, 2001. Active glycation in neurofibrillary pathology of Alzheimer disease: N(epsilon)-(carboxymethyl) lysine and hexitol-lysine. Free Radic. Biol. Med., 31: 175180 .

27. Smith, M.A., P.L. Richey Harris, L.M. Sayre, J.S. Beckman and G. Perry, 1997. Widespread peroxynitrite-mediated damage in Alzheimer's disease. J. Neurosci., 17: 2653-2657.

28. Sayre, L.M., D.A. Zelasko, P.L. Harris, G. Perry, R.G. Salomon and M.A. Smith, 1997. 4Hydroxynonenal-derived advanced lipid peroxidation end products are increased in Alzheimer's disease. J. Neurochem., 68: 20922097.

29. Honda, K., M.A. Smith, X. Zhu, D. Baus, W.C. Merrick, A.M. Tartakoff, T. Hattier, P.L. Harris, S.L. Siedlak, H. Fujioka, Q. Liu, P.I. Moreira, F.P. Miller, A. Nunomura, S. Shimohama and G. Perry, 2005. Ribosomal RNA in Alzheimer disease is oxidized by bound redox-active iron. J. Biol. Chem., 280: 20978-20986.

30. Song, M.S., I. Mook-Jung, H.J. Lee, J.Y. Min and M.H. Park, 2007. Serum anti-amyloid-beta antibodies and Alzheimer's disease in elderly Korean patients. J. Int. Med. Res., 35: 301-306.

31. Fullerton, S.M., G.A. Shirman, W.J. Strittmatter and W.D. Matthew, 2001. Impairment of the blood-nerve and blood-brain barriers in apolipoprotein e knockout mice. Exp. Neurol., 169: 13-22.

32. Piddlesden, S.J., H. Lassmann, F. Zimprich, B.P. Morgan and C. Linington, 1993. The demyelinating potential of antibodies to myelin oligodendrocyte glycoprotein is related to their ability to fix complement. Am. J. Pathol., 143: 555564.

33. He, X.P., M. Patel, K.D. Whitney, S. Janumpalli, A. Tenner and J.O. McNamara, 1998. Glutamate receptor GluR3 antibodies and death of cortical cells. Neuron, 20: 153-163.

34. Algotsson, A. and B. Winblad, 2007. The integrity of the blood-brain barrier in Alzheimer's disease. Acta Neurol. Scand., 115: 403-408. 